\begin{tabular}{|l|l|c|c|}
\hline Eiszeitalter u. Gegenwart & $\mathbf{4 6}$ & $18-31$ & Hannover 1996 \\
\hline
\end{tabular}

\title{
Zur jungholozänen Oberflächen- und Bodenentwicklung der Siegaue im Hennefer Mäanderbogen*)
}

\author{
Karl Heusch, Johannes BotscheK \& Armin SkowroneK**)
}

Late Holocene, alluvial soils, surface and soil development, Sieg river, mining, Nordrhein-Westfalen

\begin{abstract}
Kurzfassung: Die jungholozänen carbonatfreien Auenlehme im Talabschnitt der Sieg bei Hennef können nach geomorphologischen, sedimentologischen und pedologischen Untersuchungen vier Auenterrassen zugeordnet und ihre Ablagerung zeitlich eingegrenzt werden. Die Obere Auenterrasse trägt eine Auenparabraunerde-Auenbraunerde, deren Entwicklung in die ältere Eisenzeit zu stellen ist. Während sich auf der älteren Mittleren Auenterrasse eine kräftige latènezeitliche Aucnbrauncrde gebildet hat, reicht die Pedogenese auf der jüngeren Mittleren Auenterrasse nur bis zum Braunauenboden mittelalterlich/frühneuzeitlichen Ursprungs. Die jüngste, maximal 200 Jahre alte, geomorphologisch-pedologische Einheit stellt die Untere Auenterrasse dar. Auf ihr ist die Bodenentwicklung erst bis zum Auengley/Auenregosol fortgeschritten. Für die Hochflutsedimente mit Parabraunerde-Bildung auf der weichselzeitlichen Niederterrasse ist eine Ablagerung in der Bronzezeit anzunehmen. Ein vermutlich altholozäner Boden ist lediglich als Fragment, vom anthropogen bedingten Auenlehm fossiliert, auf der Niederterrasse erhalten.
\end{abstract}

\section{[Late Holocene surface and soil development of the Sieg river floodplain near Hennef]}

Abstract: The flood loams in the Sieg valley near Hennef
were investigated with geomorphological, sedimentologi-
cal and pedological methods. They are free of carbonate.
Four late Holocene floodplain terraces can be seperated
and dated. On the Upper Floodplain Terrace an Auen-
parabraunerde-Auenbraunerde (Fluvi-Luvic Cambisol) of
early Iron Age was found. The pedogenesis on the
older Middle Floodplain Terrace lead to a Typische
Auenbraunerde (Fluvi-Eutric Cambisol) of Latène Age. The
younger part of this terrace is covered by a Braunauen-
boden (Eutric Fluvisol) of late Middle Ages/modern times.
The Lower Floodplain Terrace is the youngest formation,
at most 200 years old. It is characterized by an Au-
engley/Auenregosol (Fluvi-Eutric Gleysol). The Pa-
rabraunerde (Luvisol) on the Weichselian Low Terrace is

*) Erweiterte Fassung eines Vortrages des Erstautors am 20. September 1994 in Leipzig anläßlich der 27. Jahrestagung der Deutschen Quartärvereinigung.

**) Anschrift der Verfasser: Dipl.-Ing. Agr. K. Heusch, Dr. J. Botschek und Prof. Dr. A. Skowronek, Institut für Bodenkunde der Universität, Nußallee 13, D-53115 Bonn. supposed to be of Bronze Age. That anthropogenic flood loams bury traces of a presumably early Holocene soil on the Low Terrace.

\section{Einleitung}

Die Erforschung der Ursachen holozäner Flußdynamik ist seit über 20 Jahren Ziel zahlreicher Untersuchungen. Dabei werden drei Erklärungsansätze diskutiert:

- Verstärkte Umlagerungs- und Sedimentationsprozesse werden durch Klimaschwankungen verursacht. Nach Brunnacker (1978) sind sieben „Aktivitätsphasen" am Niederrhein und nach SCHIRMER (1991a) sieben „holozäne Terrassen“ am Obermain klimagenetischen Ursprungs. Der anthropogene Einfluß ist hierbei von sekundärer Bedeutung.

- Holozäne Terrassen entstehen durch Prozesse, die von der Eigendynamik des Flusses gesteuert werden. Sie haben nach Buch (1988) an der Donau zur Ausbildung von drei „Auenstufen“ geführt. Äußere Einflüsse wie Klimaschwankungen und Landnutzungsgeschichte wirken sich allenfalls modifizierend aus.

- Erhöhte fluviale Aktivität und die Zunahme von „Auenlehm-Bildungsphasen“ sind durch menschliche Eingriffe wie Rodungen und ackerbauliche Nutzung größerer Flächen verursacht worden. Diese Ansicht wird z. B. von Wildhagen \& Meyer (1972) für das Leinetal und auch von Hiller \& LitT \& EissMANN (1991) für den Saale-Elbe-Raum vertreten.

Die holozäne Flußdynamik wird also je nach Standpunkt der Autoren vor allem mit bestimmten Klimabedingungen, mit der Eigendynamik des Flusses oder mit anthropogenen Einflüssen in Verbindung gebracht. DaLCHOW (1989) kombiniert diese Einflußgrößen und führt den jungholozänen Auenlehm überwiegend auf anthropogene Ursachen zurück. Dessen Verbreitung und die Erhaltung von mittel-/altholozänem Auenlehm macht er aber von dem - klimabedingten - Eintiefungsbetrag der Flüsse in den Talboden der kaltzeitlichen Niederterrasse abhängig. 


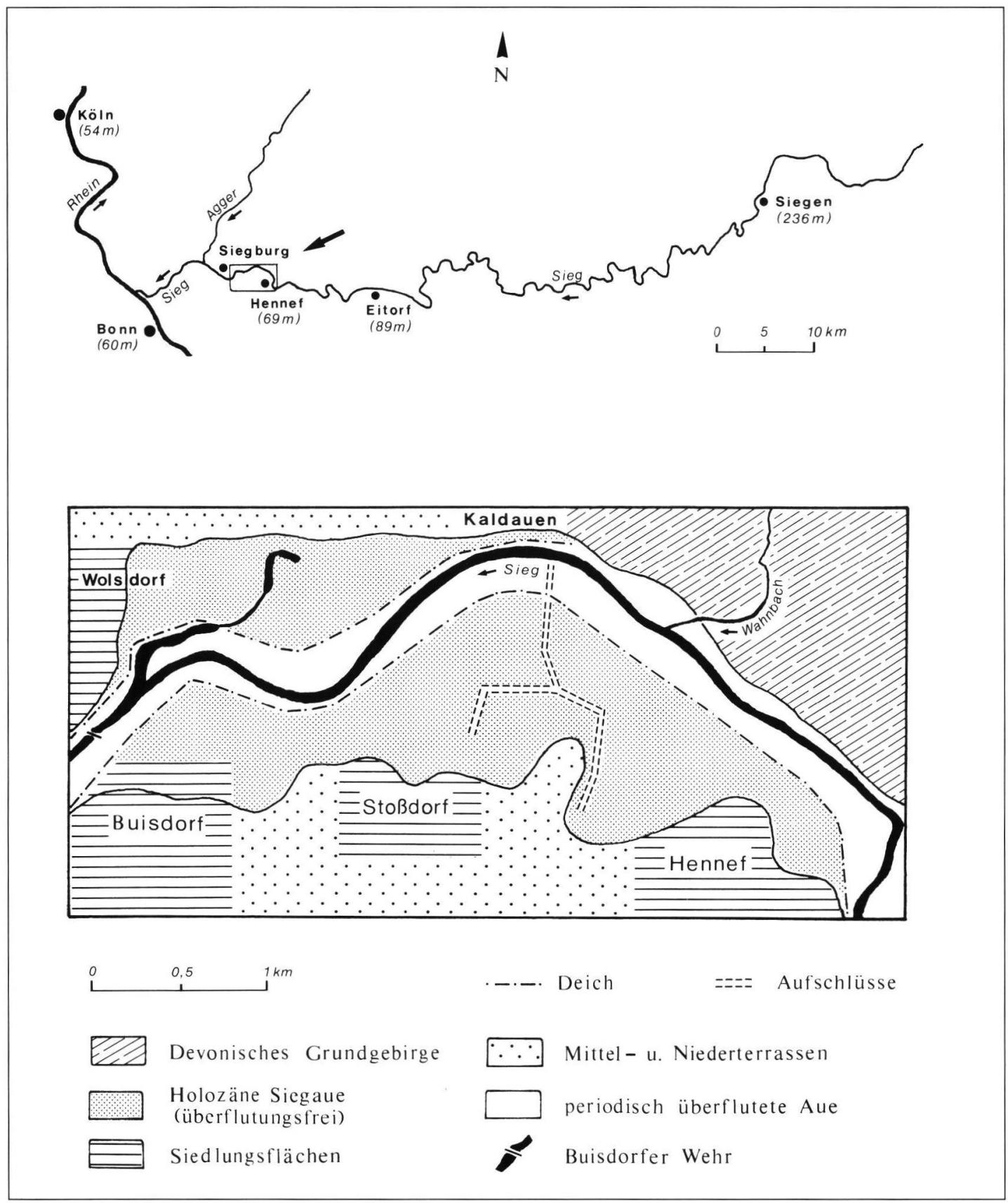

Abb. 1: Die Siegaue im Hennefer Mäanderbogen.

Fig. 1: The Sieg river floodplain near Hennef.

In diesem Beitrag wird zunächst die räumliche Verbreitung holozäner Sedimente in der Siegaue bei Hennef dargestellt. Anhand geomorphologisch-sedimentologischer und bodenkundlicher Untersuchungsergebnisse sollen dann die Einflußgrößen der jungholozänen Sedimentation bestimmt sowie eine Bodenzeitreihe (Bodenchronosequenz) erarbeitet werden.

\section{Untersuchungsraum}

Die Sieg entspringt im Rothaargebirge in $608 \mathrm{~m}$ Höhe über NN östlich von Siegen und mündet nach $144 \mathrm{~km}$ Lauflänge nördlich von Bonn-Beuel in den Rhein (s. KREMER \& CaSpers 1986: 3-4, u. hier Abb. 1). In ihrem Ober- und Mittellauf durchschneidet 
sie kalkfreie unterdevonische Schichten aus Tonschiefer, Grauwacken und Sandsteinen (Gramsch 1978: 44). Während der windungsreiche Lauf des Siegtales, das erstmals von KNUTH (1923) geomorphologisch untersucht wurde, in seinem mittleren Abschnitt überwiegend tektonisch vorgezeichnet ist, kann der Fluß nach seinem Eintritt in die Niederrheinische Bucht bei Hennef in großen Bögen bis zur - 12 Stromkilometer entfernten - Mündung in den Rhein frei mäandrieren.

Das Untersuchungsgebiet befindet sich im südöstlichen Teil der Niederrheinischen Bucht, in der sog. Siegburger Bucht bei Hennef (Abb. 1). Hierbei handelt es sich um eine mit tertiären Tonen und quartären Schottern der Sieg aufgefüllte Senkungszone, die tektonisch der Kölner Scholle zuzuordnen ist (E. Schröder 1969, Behl \& Krämer 1992). E. SchröDER (1965: 43) erkannte bei Hennef eine schwache Schollenkippung in Richtung NNE, die nach Gramsch (1978: 62) eine stetige Verlagerung der Sieg nach Norden bis in die Gegenwart bewirkte. Tatsächlich ist hier fast die gesamte Aue nur südlich der Sieg ausgebildet, deren Grenze zur Niederterrasse auf der Linie Buisdorf-Stoßdorf-Hennef verläuft. Im N und NE wird die Siegaue durch den weitgespannten Mäanderbogen vor den steil ansteigenden Hängen des Bergischen Landes begrenzt.

Die Höhe der weichselzeitlichen Niederterrasse beträgt bei Hennef $69 \mathrm{~m}$ über NN und sinkt bei Buisdorf auf $62 \mathrm{~m}$ über NN ab. Eine deutliche Geländekante bildet den Übergang zur Talaue, die im allgemeinen nur 2 bis $4 \mathrm{~m}$ unter dem Niveau der Niederterrasse liegt. Während die laterale Ausdehnung der Siegaue in ihrem Mittellauf wenige $100 \mathrm{~m}$ beträgt, verbreitert sie sich mit dem Eintritt in die Siegburger Bucht auf 1 bis $1,5 \mathrm{~km}$. Ein in den $30 \mathrm{er}$ Jahren fertiggestellter Deich folgt dem aktuellen Flußlauf durch das gesamte Untersuchungsgebiet und verengt die hochwasserführende Sieg auf durchschnittlich $200 \mathrm{~m}$ Breite.

\section{Geomorphologisch-sedimentologische Einheiten}

Die geologische Aufnahme 1:25 000 Blatt 5209 Siegburg aus dem Jahre 1935 unterscheidet im Auenbereich der Sieg bereits zwei Niveaus. Auf der Karte wird die Siegaue von zahlreichen Rinnen („Talboden des Siegtales“) in inselartige Flächen („Höhere Talstufe oder Inselterrasse") gegliedert (UDLuft 1977: 33). Udulft wie auch KNuTH (1923) kennzeichnen mit der sog. „Inselterrasse“ aber nicht eine selbständige nacheiszeitliche Aufschüttung, sondern eine Erosionsform in der weichselzeitlichen Niederterrasse. Untersuchungen insbesondere von Schirmer (1983a) haben inzwischen gezeigt, daß eine „Inselterrasse“ im o. a. Sinne einen Terrassenkörper beschreibt, der aus mehreren, unterschiedlich alten, im Zuge der Mäanderbildung entstandenen, holozänen Sedimenten zusammengesetzt ist. Solche, oft höhengleiche Terrassen können anhand von Rinnensystemen und ihren Ablagerungen nach ScHIRMER (1983a) in Reihenterrassen gegliedert werden. Dabei wird das Netz der Auenrinnen (Altarme, Mäanderbögen), ihr morphologischer Relieftyp und das gegenseitige Abschneiden von Auenrinnensystemen durch Nahtrinnen (geomorphologische Diskordanzen) zur Trennung verschieden alter spätglazialer und holozäner Auenterrassen genutzt.

Im Arbeitsgebiet erweist sich dieser geomorphologische Ansatz als problematisch. Hier ergab sich bei breiten und lang durchziehenden Rinnen z. B. die Schwierigkeit, Nahtrinnen von Auenrinnen zu unterscheiden. Mit Ausnahme des großen Mäanderbogens zwischen Kaldauen und Wolsdorf sind an der Oberfläche auch keine weiteren Mäander sichtbar. Zudem ist durch die ackerbauliche Nutzung fast aller innendeichs gelegenen Flächen das typische Gleithang/Prallhang-Relief weitgehend eingeebnet worden und ein Auskartieren von Reihenterrassengrenzen schwierig. Einen guten Einblick in den stratigraphischen Aufbau der Aue ermöglichten aber 3,5 m tiefe, die gesamte Aue querende Aufschlüsse, die für die Verlegung einer Trinkwasserleitung angelegt wurden (Abb. 1). An diesen Profilschnitten in S-N- und E-W-Richtung wurden umfassende sedimentologische und bodenkundliche Daten erhoben.

\subsection{Weichselzeitliche Niederterrasse}

Die kaltzeitliche Niederterrasse ist bis auf ein kleines Vorkommen bei Kaldauen nur südlich der Sieg erhalten. Hier bildet sie eine große zusammenhängende Fläche, die sich von Hennef über Stoßdorf bis Buisdorf in zunehmender Breite nach W erstreckt. Das Gefälle der Niederterrasse beträgt zwischen Hennef und Buisdorf ca. 1,5\%. Im Mündungsbereich der Sieg kommt es sogar zu einer Gefälleumkehrung, so daß der Niveauabstand zwischen den holozänen Auenterrassen und der weichselzeitlichen Niederterrasse mit 7 bis $10 \mathrm{~m}$ wesentlich größer ist als im Untersuchungsgebiet. GramsCH (1978: 64) erklärt diesen Reliefunterschied damit, daß die Sieg in der Nähe ihrer Erosionsbasis (Rhein) die Schotter im Auenbereich verstärkt umgelagert und ausgeräumt hat, als sich der Rhein während des Holozäns in sein Hochflutbett eintiefte. Der geringe Niveauabstand zwischen Niederterrasse und Talaue bei Hennef führte indes zu Überflutungen der gesamten Niederterrassenfläche. Die Sedimente der Hochflutlehmdecke auf der Niederterrasse 
mit durchschnittlichen Mächtigkeiten von 1,0 - 1,5 m, die Udluft (1977: 31) in dieselbe Ablagerungszeit stellt wie die der Lehme in der Talaue, zeugen davon. WARSTAT (1981) findet am Terrassenrand sogar noch größere Mächtigkeiten vor. Selbst im Mündungsgebiet greifen die Hochflutlehme der Talaue noch randlich auf die Niederterrasse über (THOste 1974). So wird auch die Fundstelle einer jungneolithisch/bronzezeitlichen Siedlungsstätte in Niederpleis (2 Stromkilometer talwärts) mit zahlreichen Steinbeilen von mehreren Metern dickem Auenlehm überdeckt (MARSCHAll \& NARR \& v.USLAR 1954: 130). Die Siedlungsstelle befand sich $20 \mathrm{~m}$ von der Terrassenkante entfernt direkt auf der Schotteroberfläche der Niederterrasse. Die Körnung des Hochflutlehms, der bis zum Schotter holzkohleführend ist, wechselt mit zunehmender Tiefe von schluffigem Lehm zu lehmigem Sand. Oft finden sich im Liegenden der Sande wieder feinkörnigere Sedimente.

\subsection{Holozäne Auenterrassen}

Als holozäne Auenterrassen werden zunächst drei Terrassen(treppen) unterhalb des Niveaus der weichselzeitlichen Niederterrasse unterschieden: Obere, Mittlere und Untere Auenterrasse (Abb. 2 u. 3). Die in den Aufschlüssen sichtbaren Schotter konnten anhand ihrer sedimentologischen Merkmale (nach SCHIRMER 1983a) deutlich als holozäne Umlagerungsprodukte eines mäandrierenden Flußlaufes mit Nebenrinnen erkannt werden. KLOSTERMAN (1992: 175) bezeichnet solche Abflußsysteme mit einem gewundenen Hauptarm und kleineren Seitenarmen am Niederrhein als „Furkationstyp“. Leider war durch die geringe Tiefe der Aufschlüsse die Diskordanz zwischen Ablagerungen der Niederterrasse und Umlagerungen des Holozäns an keiner Stelle erkennbar. Rinnenfüllungen von maximal 4,5 $\mathrm{m}$ Mächtigkeit lassen auf eine entsprechende Umlagerungstiefe der Schotter aller holozänen Auenterrassen schließen. Die drei, mit jeweils 0,5 bis $1,0 \mathrm{~m}$ Höhenunterschied treppenartig voneinander abgesetzten Auenterrassen sind mit einem relativ geringen Eintiefungsbetrag von 2 bis $4 \mathrm{~m}$ - in die Ablagerungen der Niederterrasse eingeschachtelt. Die Hochflutlehme der Auenterrassen sind ebenfalls mit fein verteilten Holzkohleresten durchsetzt.

\section{Obere Auenterrasse}

Die Obere Auenterrasse stellt mit 1,5 bis 2,5 m über bordvollem Siegwasserspiegel das höchstgelegene Niveau in der Siegaue dar. Von der Niederterrasse ist diese Stufe durch eine 1,5 bis $2 \mathrm{~m}$ hohe Geländekante abgesetzt. Ihre Verbreitung beschränkt sich auf zwei Flächen bei Zissendorf, die bogenförmig in die Niederterrasse eingelassen sind. Die Mächtigkeit der Hochflutsedimente, die durch eine nach oben abnehmende Korngröße von stark schluffiglehmigem Sand bis zu schluffigem Lehm gekennzeichnet sind, variiert zwischen 0,9 und $1,5 \mathrm{~m}$.

\section{Mittlere Auenterrasse}

Die Mittlere Auenterrasse nimmt den größten Teil der Siegaue ein. Sie besitzt eine relative Höhe von 1-1,5 m über bordvollem Siegabfluß. Von der Oberen Auenterrasse ist sie durch eine stark ausgeprägte Rinne und einen niedrigen Geländeanstieg (ca. $0,5 \mathrm{~m})$ getrennt. Nur östlich von Zissendorf bildet eine deutliche Kante die Grenze zu der hier knapp $1 \mathrm{~m}$ höher gelegenen Oberen Auenterrasse. In Bereichen, wo die Mittlere Auenterrrasse direkt an die Niederterrasse stößt (z. B. bei Zissendorf), liegen zwischen beiden Niveaus 2,5 bis $3 \mathrm{~m}$. Das Gefälle der Mittleren Auenterrasse ist geringer als das der Niederterrasse. Es erreicht zwischen Buisdorf und Pegel Kaldauen 1,3\%0 und verringert sich bis Hennef auf 1,1\%. Die Mächtigkeit der feinklastischen Sedimente wechselt kleinräumig sehr stark; in Rinnen können sogar über $4 \mathrm{~m}$ erreicht werden. Die durchschnittliche Dicke des Auenlehms auf der Mittleren Auenterrasse liegt bei ca. $1 \mathrm{~m}$, wobei aber auch - z.T. flächenhaft - bis zu $2 \mathrm{~m}$ mächtiger lehmiger Schluff bis schluffiger Lehm abgelagert worden ist.

\section{Untere Auenterrasse}

Die Ausdehnung der jüngsten Terrasse läßt sich auf weite Strecken durch eine 1-1,5 $\mathrm{m}$ hohe morphologische Kante von der Mittleren Auenterrasse abtrennen. Sie begleitet als schmales, meist weniger als $50 \mathrm{~m}$ breites Band den aktuellen Flußlauf. Zur jüngsten Terrasse gehört auch die Umlauffläche des großen Mäanders zwischen Kaldauen und Wolsdorf. Das Gefälle der Terrasse entspricht mit durchschnittlich 1,2\%o ungefähr dem der heutigen Sieg. Nur im Rückstaugebiet des Buisdorfer Wehres kommt es zu einer starken Reduzierung auf 0,2 bis $0,3 \%$ Die Körnung der sehr feingeschichteten, durchschnittlich $1 \mathrm{~m}$ mächtigen Auenlehmsedimente der Unteren Auenterrasse variiert zwischen kiesigem Sand und schluffigem Lehm. Sie weisen oft hohe Humusgehalte auf.

\subsection{Auenrinnen und Sedimente}

Morphologisch stark ausgeprägte Rinnen und Geländekanten bilden die Grenzen aller oben beschriebenen Terrassen der Siegaue (Abb. 2 u. 3). Tiefgründige, sehr feinkörnige Auenrinnensedimente sind basal z.T. holz- und/oder pollenführend, sie können somit für absolute Datierungen $\left({ }^{14} \mathrm{C}\right)$ und zur Rekonstruktion der Vegetation herangezogen werden. Allerdings lassen die hier 


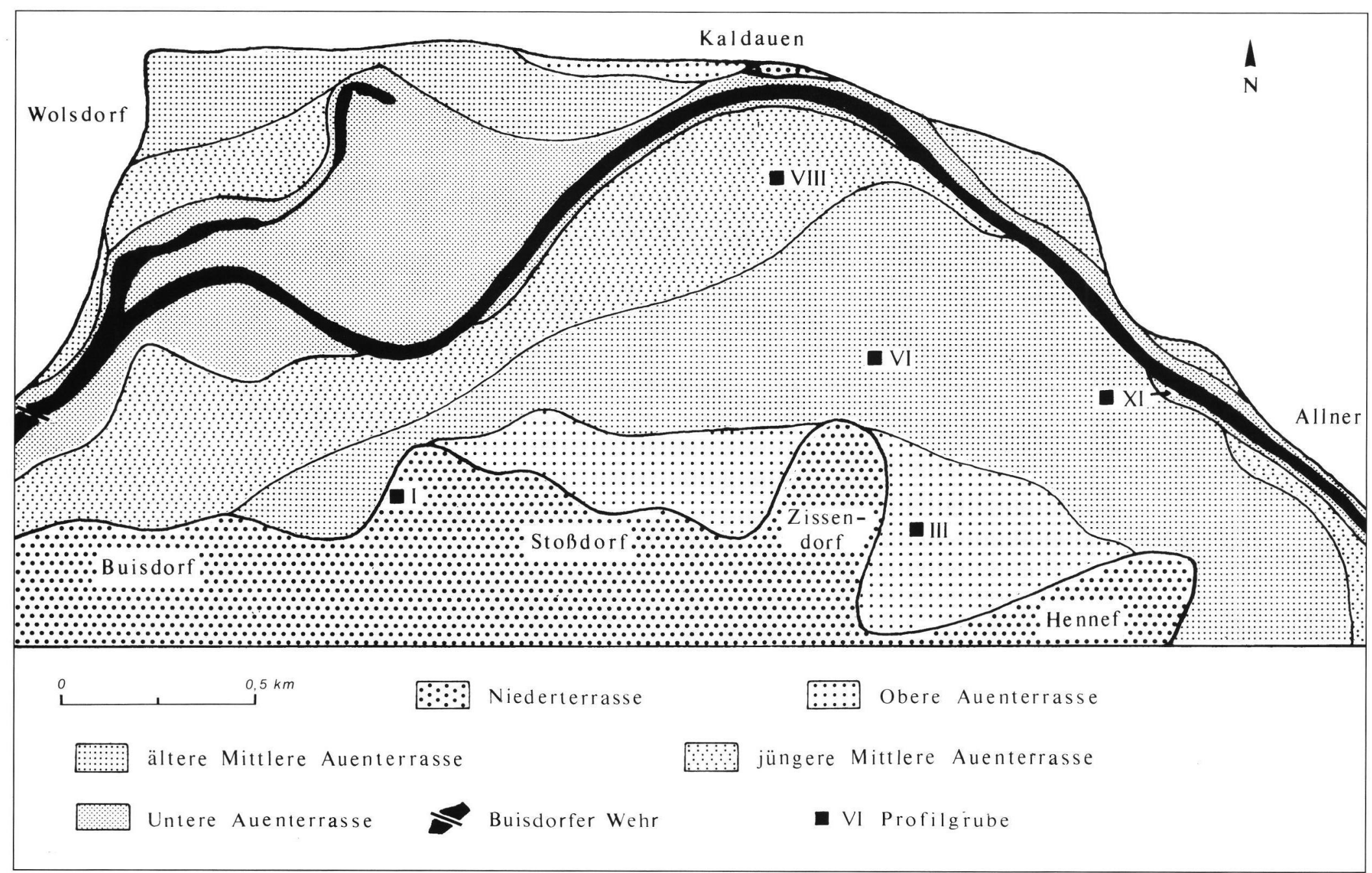

Abb. 2: Stratigraphische Gliederung der Sieg-Auenterrassen Fig. 2: Stratigraphic division of Sieg river alluvial terraces 


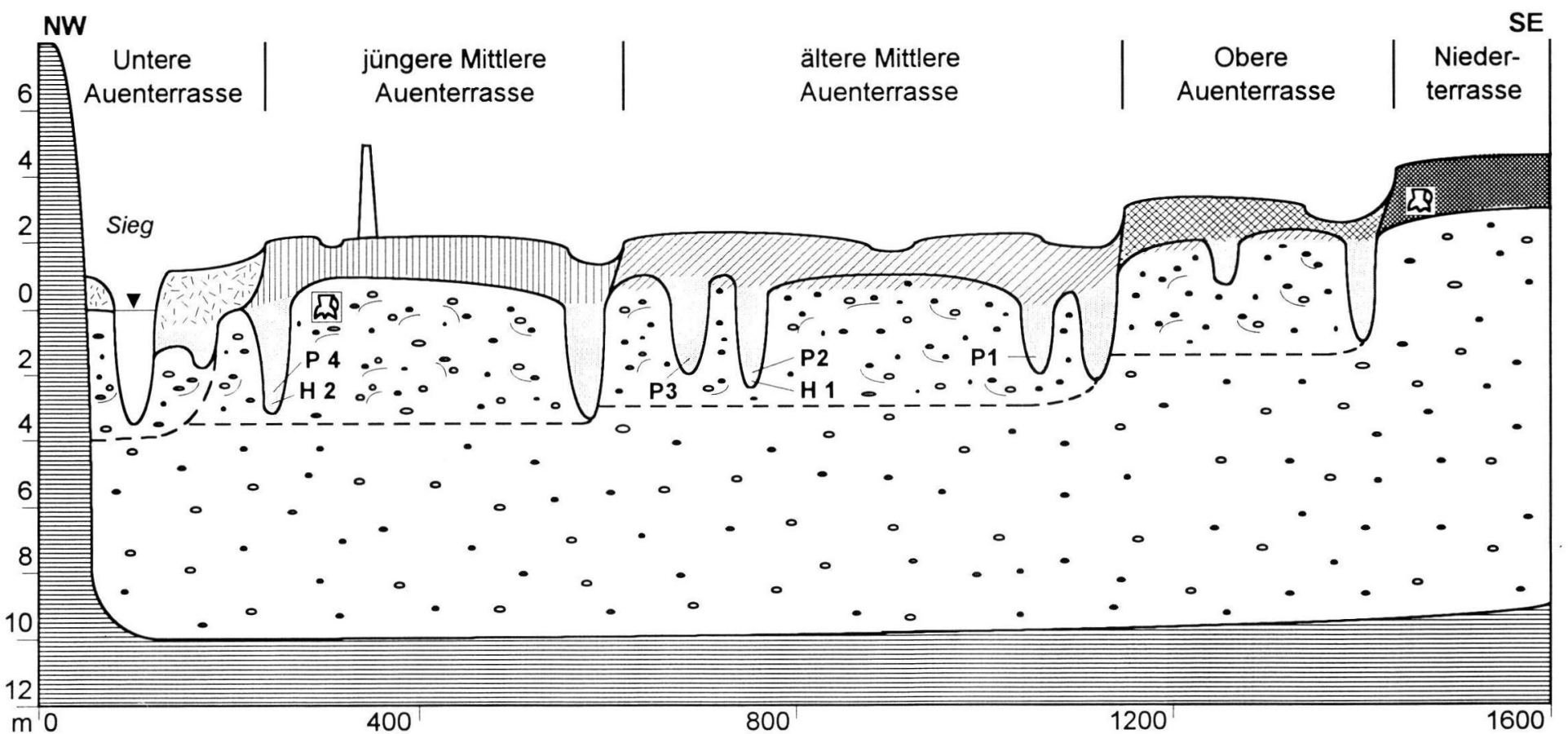

\begin{tabular}{llll}
\hline Parabraunerde & Auenparabraunerde-Auenbrau \\
\hline B. & Auengley / Auenregosol \\
\hline P, H & Polozäne Schotter & pleistozäne Schotter \\
\hline
\end{tabular}

Auenbraunerde

feinkörnige Rinnenfüllung

devonisches Grundgebirge 
Tab. 1: Pollenanalyse von Rinnensedimenten der Siegaue

Tab. 1: Pollen analysis, of channel sediments of Sieg river floodplain

\begin{tabular}{|c|c|c|c|c|}
\hline $\begin{array}{l}\text { Pollenprobe } \\
\text { Fundstelle } \\
{ }^{14} \text { C-Alter der Holzproben }\end{array}$ & P 1 & $\begin{array}{l}\text { P } 2 \\
\text { MAT } \\
\pm 70 \\
\end{array}$ & P 3 & $\begin{array}{c}\text { P } 4 \\
\text { jMAT } \\
800 \pm 50 \mathrm{BP} \\
\end{array}$ \\
\hline & \multicolumn{4}{|c|}{ [in \% der Gesamtpollensumme] } \\
\hline Baumpollen & 47 & 43 & 40 & 58 \\
\hline Nichtbaumpollen & 53 & 57 & 60 & 42 \\
\hline Wald & \multicolumn{4}{|c|}{ [in \% der Baumpollensumme] } \\
\hline Pinus & 2 & 0 & 3 & 5 \\
\hline Picea & 0 & 0 & 0 & 0 \\
\hline \multicolumn{5}{|l|}{ Eichenmischwald: } \\
\hline Querc., Tilia, Acer, Fraxin., Ulm. & 30 & 20 & 30 & 17 \\
\hline Fagus & 7 & 11 & 27 & 32 \\
\hline Carpinus & 7 & 8 & 11 & 16 \\
\hline Castanea & 0 & 0 & 0 & 1 \\
\hline Alnus * & 65 & 121 & 256 & 158 \\
\hline \multicolumn{5}{|l|}{ Sonstige Gehölze } \\
\hline Salix, Betula, Corylus & 54 & 61 & 29 & 29 \\
\hline Wasserpflanzen & \multicolumn{4}{|c|}{ [in \% der Nichtbaumpollensumme] } \\
\hline Potagometon, Myriophyll., Typha & 1 & 0 & 1 & 0 \\
\hline Pediastrum & 0 & 1 & 0 & 0 \\
\hline \multicolumn{5}{|l|}{ Feuchtwiesen } \\
\hline Filipendula, Mentha, Lotus & 4 & 0 & 1 & 9 \\
\hline \multicolumn{5}{|l|}{ Gräser } \\
\hline Poaceae & 39 & 28 & 46 & 35 \\
\hline Cereales & 12 & 5 & 7 & 13 \\
\hline \multicolumn{5}{|l|}{ Ruderale } \\
\hline Artemisia, Chenopod., Polygon. & 15 & 29 & 15 & 13 \\
\hline Brassicaceae & 1 & 3 & 3 & 0 \\
\hline Asteraceae & 9 & 22 & 11 & 7 \\
\hline Sonstige & 19 & 12 & 16 & 23 \\
\hline
\end{tabular}

* Alnus geht als lokaler, großer Pollenspender nicht in die Baumpollensumme ein, wird aber auf diese bezogen.

erhobenen Einzeldaten nur bedingt Aussagen über die Ablagerungszeit zu. Nach Schirmer (1983b) können einerseits die basalen Rinnenfüllungen so alt wie die Terrasse selbst sein. In diesem Fall stellen die Rinnensedimente einen „terminus ab quo“ für die Terrasse dar. Andererseits werden Auenrinnen noch lange Zeit von Hochwässern benutzt. Dabei können ältere Sedimente ausgeräumt und die Rinnen neu verfüllt werden. Man erhält dann einen „terminus post quem“, der erheblich jünger sein 
kann als die Anlage des Terrassenkörpers selbst. Dies muß im Untersuchungsgebiet mitberücksichtigt werden, da außergewöhnliche Hochwässer bis 1890 immer wieder die Niederterrassenkante erreicht haben (Archiv des Rhein-Sieg-Kreises 1976). Auf der Niederterrasse zeigen sich westlich von Stoßdorf wenige, breite $(50-60 \mathrm{~m})$ und flache $(<0,5$ m) Rinnen, die keine Aussagen über den Gerinnetyp zulassen. Unterhalb von Stoßdorf wird die Obere Auenterrasse von einigen $\max .30 \mathrm{~cm}$ tiefen und 30 bis $40 \mathrm{~m}$ breiten sekundären Auenrinnen durchzogen. Östlich von Zissendorf bildeł eine ca. $50 \mathrm{~m}$ breite und bis zu $1 \mathrm{~m}$ tiefe Erosionsrinne einen nach NE geöffneten Halbkreis. Keine der erwähnten Rinnen ist holz- oder pollenführend, da ihnen in der Regel die Füllung mit feinkörnigem Sediment fehlt.

Das Relief der Mittleren Auenterrasse wird von zahlreichen flachgründigen Rinnen bestimmt, die sich mit zunehmender Nähe zur Sieg dem aktuellen Verlauf angleichen. Auffällig ist, daß trotz der deutlich im Gelände auskartierbaren Rinnen keine stärker gekrümmten Bögen oder längere Bogenstücke, die auf abgeschnittene Mäander hindeuten könnten, sichtbar sind. Vielmehr sind durch Hochwassereinwirkung und die damit einhergehende rückschreitende Erosion Mäanderreste und andere Hohlformen und Rinnen verbunden worden. Dies erklärt auch, warum die größeren, lang durchziehenden Rinnen fast ausnahmslos dem Hochwasserabfluß in E-W-Richtung, wie er vor dem Deichbau existierte, folgen. Schließlich bleibt zu berücksichtigen, daß sich die Sieg im Holozän zumindest zeitweise in einem Übergangsstadium zwischen verwildertem (braided river) und mäandrierendem Strom (Furkationstyp) befand, so daß verlandete bogenförmige Altarme nicht unbedingt zu erwarten sind. Selbst für die heutige Sieg zwischen Hennef und Siegburg besteht aufgrund des relativ großen Gefälles von durchschnittlich 1,2\% bei erhöhtem Abfluß zumindest theoretisch die Möglichkeit, das Gerinnebettmuster in Richtung eines verzweigten Flußtyps zu verändern.

Die morphologisch gut sichtbaren Rinnen der Mittleren Auenterrasse sind oft nur geringmächtig mit umgelagertem Boden gefüllt (vgl. WARSTAT 1985), was auf sekundäre Auenrinnen hinweist. Durch die Aufschlüsse wurden jedoch einige verdeckte bis zu $4,5 \mathrm{~m}$ tiefe Rinnen angeschnitten, die basal mit blauschwarzem tonigem und holzführendem Sediment gefüllt sind. Die Ergebnisse der aus diesen Sedimenten gewonnenen zwei ${ }^{14} \mathrm{C}$ - und 4 Pollenproben sind in Tab. 1 zusammengefaßt, die Fundstellen sind der Abb. 3 zu entnehmen.

Die hohe Anzahl an lokalem Alnus sowie das Vorhandensein von Cereales und inkohlter organischer Substanz in fast allen Fundstellen mögen davon zeugen, daß z.Zt. ihrer Sedimentation die Siegaue teilweise unter Kultur stand. In den Proben 1-3, die alle den älteren Rinnen entnommen sind (wenn man von einer kontinuierlichen Flußverlagerung nach Norden ausgeht), überwiegen die Nichtbaumpollen neben z.T. hohen prozentualen Anteilen an Alnus, Carpinus und Quercus. Das Pollenspektrum deutet auf eine vorwiegend offene Vegetation mit Erlen-Bruchwald-Beständen an den Wasserläufen und vereinzelten Eichenmischhainen zwischen den Wirtschaftsflächen. Die Holzprobe 1, die aus demselben basalen Rinnensediment aus 3,5 m Tiefe gezogen wurde wie die Pollenprobe 2, weist ein ${ }^{14} \mathrm{C}$-Alter von $2210 \pm 70$ BP (HAM 3307) auf. $\mathrm{Zu}$ diesem Zeitpunkt muß die Sedimentation großer Auenlehmbereiche der südlichen Mittleren Auenterrasse weitgehend abgeschlossen gewesen sein. Die Vegetation und das ${ }^{14} \mathrm{C}$-Alter entspricht somit der für die westliche Niederrheinische Bucht festgestellten "Quercus-NBP-Zone" nach KaLIS \& Meurers-Balke (1994), die diese in eine Zeit um 2700-1700 BP stellen. Ihre archäobotanischen Befunde zeigen nicht nur eine starke Entwaldung, sondern belegen für die späte Eisenzeit (ab $250 \mathrm{v}$. Chr.) eine zunehmende Intensivierung der Landwirtschaft.

In der Probe 4 aus einer jüngeren Rinne der Mittleren Auenterrasse überwiegen hingegen die Baumpollenkörner mit erhöhten Werten an nicht lokalem Pinus. Erstmals tritt hier auch die mit den Römern eingewanderte Castanea auf. Dieses Pollenspektrum weist auf die im Spätmittelalter verbreiteten Flurwüstungen hin. Die zweite Holzprobe besitzt ein ${ }^{14} \mathrm{C}$-Alter von $800 \pm 50 \mathrm{BP}$ (HAM 3308) und kündigt somit für die Auenlehme der nördlichen Mittleren Auenterrasse eine mittelalterliche Entstehung an. Die am Rand zur Unteren Auenterrasse gelegene Auenrinne ist aber z.T. mit Schottern überlagert. Im Aufschluß war neben dieser Rinne eine Kiesbank angeschnitten, in der in $2 \mathrm{~m}$ Tiefe unter Geländeoberkante eine Scherbe aus dem 15. Jahrhundert gefunden wurde. Unmittelbar neben der Holzprobe in sandig-kiesiger Lage erfolgte ein weiterer Keramikfund ebenfalls aus dem 15. Jahrhundert. Vermutlich hat die Sieg bei ihrer Laufverlegung nach Norden immer wieder größere Kiesbänke aufgeschüttet, wobei südlich gelegene Altarme z.T. nachträglich überdeckt wurden. Die Sedimentation dieser Auenterrasse war daher erst in der frühen Neuzeit abgeschlossen.

Auf der Unteren Auenterrasse sind noch drei Auenrinnen erhalten, die die sinoidale Bewegung des großen, teilweise verlandeten Mäanders zwischen Kaldauen und Wolsdorf dokumentieren. Die sehr große Amplitude des Mäanderbogens steht im Zusammenhang mit dem Buisdorfer Wehr, das einen $2 \mathrm{~km}$ langen Rückstau und damit eine starke 
Verringerung des Gefälles verursachte. Das Siegwehr, das erstmals 1391 urkundlich erwähnt wird (Wisplinghoff 1964), liegt spätestens seit der Mitte des 18. Jahrhunderts an der heutigen Stelle.

\section{Pedostratigraphie und Bodenentwicklungsstadien}

Die Ergebnisse der geomorphologisch-sedimentologischen Untersuchungen zur holozänen Talgeschichte der Sieg können durch die Kenntnis der Auenbodenentwicklung überprüft und ergänzt werden. Da die Ausprägung pedogenetischer Merkmale auch von der Dauer der Bodenbildung abhängt, müßten unterschiedlich alte Terrassen demnach charakteristische Böden tragen; eine Abfolge von Reihenterrassen würde dann eine Chronosequenz oder Bodenzeitreihe enthalten (vgl. D. SCHRÖDER 1979). Die Tatsache, daß sich in gleichartigen Flußsedimenten unterschiedliche Bodentypen entwickelt haben, ermöglicht eine relative Alterseinstufung der Auenterrassen.

\subsection{Bodenchronosequenz}

In der Auenlehmdecke der weichselzeitlichen Niederterrasse, die nur in ihrem Randbereich untersucht worden ist, hat sich eine Parabraunerde entwickelt. Der Tonanreicherungshorizont (Bt) in Profil I enthält mit über $25 \%$ die höchsten Tonge-

Tab. 2: Korngrößenverteilung, organische Substanz und pH-Wert der Auenböden

Tab. 2: Particle size distribution, organic matter and $\mathrm{pH}$ value of alluvial soils

\begin{tabular}{|c|c|c|c|c|c|c|c|}
\hline Bodentyp & Horizont & $\begin{array}{r}\text { Tiefe } \\
{[\mathrm{cm}]}\end{array}$ & $\mathbf{S}$ & $\begin{array}{c}U \\
{[\%]}\end{array}$ & $\mathrm{T}$ & $\begin{array}{l}\mathrm{C}_{\text {org }} \\
\text { [\%] }\end{array}$ & $\begin{array}{c}\mathrm{pH} \\
{\left[\mathrm{CaCl}_{2}\right]}\end{array}$ \\
\hline Parabraunerde & Ap & $0-33$ & 25,5 & 54,9 & 19,6 & 1,1 & 6,9 \\
\hline \multirow[t]{5}{*}{ (Profil I) } & $\mathrm{Bt}$ & $33-42$ & 33,0 & 41,4 & 25,6 & 0,4 & 6,8 \\
\hline & $\mathrm{Bv}$ & $42-71$ & 38,3 & 43,9 & 17,8 & 0,2 & 6,8 \\
\hline & IIBv & $71-92$ & 56,3 & 27,9 & 15,8 & 0,2 & 6,6 \\
\hline & IIIBv & $92-102$ & 67,7 & 20,0 & 12,3 & 0,2 & 6,8 \\
\hline & IVSBV & $102-109$ & 31,6 & 51,6 & 16,8 & 0,2 & 6,6 \\
\hline Auenparabraunerde - & Ap & $0-33$ & 16,6 & 65,3 & 18,1 & 0,9 & 6,6 \\
\hline Auenbraunerde & AlBv & $33-45$ & 15,4 & 64,6 & 20,0 & 0,5 & 6,4 \\
\hline \multirow[t]{3}{*}{ (Profil III) } & Btv & $45-65$ & 17,6 & 58,2 & 24,2 & 0,4 & 6,1 \\
\hline & IIBv & $65-75$ & 33,5 & 44,4 & 22,1 & 0,4 & 6,3 \\
\hline & IIIBv & $75-95$ & 41,6 & 38,0 & 20,4 & 0,4 & 6,3 \\
\hline Typ. Auenbraunerde & Ap & $0-34$ & 22,7 & 61,0 & 16,3 & 0,8 & 6,0 \\
\hline \multirow[t]{2}{*}{ (Profil VI) } & Bv & $34-60$ & 24,9 & 55,7 & 19,4 & 0,4 & 5,7 \\
\hline & $\mathrm{aM}$ & $60-75$ & 39,3 & 44,4 & 16,3 & 0,4 & 5,9 \\
\hline Braunauenboden & Ap & $0-32$ & 36,6 & 51,0 & 12,4 & 0,9 & 5,2 \\
\hline \multirow[t]{2}{*}{ (Profil VIII) } & aMBv & $32-65$ & 34,4 & 54,2 & 11,4 & 0,4 & 5,4 \\
\hline & $\mathrm{aM}$ & $65-89$ & 42,3 & 47,3 & 10,4 & 0,4 & 5,3 \\
\hline Auengley - & $\mathrm{Ah}$ & $0-15$ & 28,1 & 52,4 & 19,5 & 3,0 & 5,5 \\
\hline Auenregosol & aMAh & $15-25$ & 41,6 & 44,0 & 14,4 & 1,8 & 5,5 \\
\hline \multirow[t]{2}{*}{ (Profil XI) } & $\mathrm{aM}$ & $25-50$ & 61,9 & 29,3 & 8,8 & 0,5 & 5,6 \\
\hline & aMGo & $50-80$ & 70,0 & 22,9 & 7,1 & 0,2 & 5,6 \\
\hline
\end{tabular}


halte aller untersuchten Horizonte (Tab. 2). Der Lessivierungshorizont ( $\mathrm{Al}$ ) des unmittelbar am Niederterrassenrand entwickelten Bodens ist bereits durch Erosion abgetragen worden und daher nicht mehr nachweisbar. Im Profil wurde unterhalb der nach unten gröber werdenden Sande ein $7 \mathrm{~cm}$ mächtiger, stark hydromorpher Horizont (IVSBv) mit feinerer Bodenart festgestellt. Der diskordant darüberliegende sandige, dunkel erscheinende IIIBvHorizont enthält möglicherweise Reste eines begrabenen Oberbodens an der Basis der neuen Hochflutsedimentation. Ein hier zu erwartender Anstieg der organischen Substanz im Profil wird durch die erwähnte Holzkohle überlagert. Vermutlich stellt die beschriebene Diskordanz die Grenze zwischen altholozänem und mittel-/jungholozänem Auenlehm dar. Die Bodenentwicklung der Oberen Auenterrasse ist bis zu einer AuenparabraunerdeAuenbraunerde fortgeschritten. In Profil III ist der Tongehaltsunterschied zwischen dem Alund dem Bt-Horizont mit 4,2 \% nur schwach ausgebildet, was als beginnende Lessivierung gedeutet werden kann (Tab. 2). Deshalb wurden die entsprechenden Horizonte als AlBv bzw. Btv angesprochen. Das Vertikalprofil ist durch eine ausgeprägte, nach oben feiner werden-

Tab. 3: Eisengehalte und Kationenaustauschkapazität der Auenböden

Tab. 3: Iron content and cation exchange capacity of alluvial soils

\begin{tabular}{|c|c|c|c|c|c|c|}
\hline Bodentyp & Horizont & $\begin{array}{r}\text { Tiefe } \\
{[\mathrm{cm}]}\end{array}$ & $\begin{array}{c}\mathrm{Fe}_{\mathrm{d}} \\
{[\mathrm{g} / \mathrm{kg}]}\end{array}$ & $\begin{array}{c}\mathrm{Fe}_{\mathrm{t}} \\
{[\mathrm{g} / \mathrm{kg}]}\end{array}$ & $\mathrm{Fe}_{\mathrm{d}} / \mathrm{Fe}_{\mathrm{t}}$ & $\begin{array}{r}\text { KAK } \\
{\left[\mathrm{mmol}_{\mathrm{c}} /\right.} \\
100 \mathrm{~g} \text { Ton }]\end{array}$ \\
\hline Parabraunerde & $A p$ & $0-33$ & 17,39 & 33,57 & 0,52 & 39,89 \\
\hline \multirow[t]{5}{*}{ (Profil I) } & $\mathrm{Bt}$ & $33-42$ & 20,34 & 39,72 & 0,51 & 35,02 \\
\hline & Bv & $42-71$ & 19,21 & 40,81 & 0,47 & 44,65 \\
\hline & IIBv & $71-92$ & 21,40 & 47,83 & 0,45 & 43,91 \\
\hline & IIIBv & $92-102$ & 23,63 & 52,58 & 0,45 & 45,06 \\
\hline & IVSBV & $102-109$ & 18,25 & 39,60 & 0,46 & 49,23 \\
\hline Auenparabraunerde- & Ap & $0-33$ & 15,46 & 31,89 & 0,48 & 35,03 \\
\hline Auenbraunerde & AlBv & $33-45$ & 16,82 & 32,25 & 0,52 & 33,72 \\
\hline \multirow[t]{3}{*}{ (Profil III) } & Btv & $45-65$ & 21,72 & 40,03 & 0,54 & 31,76 \\
\hline & IIBv & $65-75$ & 22,58 & 42,11 & 0,54 & 34,14 \\
\hline & IIIBv & $75-95$ & 21,81 & 42,79 & 0,51 & 34,51 \\
\hline Typ. Auenbraunerde & Ap & $0-34$ & 16,66 & 29,84 & 0,56 & 29,61 \\
\hline \multirow[t]{2}{*}{ (Profil VI) } & $\mathrm{Bv}$ & $34-60$ & 20,08 & 36,87 & 0,54 & 27,54 \\
\hline & $\mathrm{aM}$ & $60-75$ & 19,82 & 37,49 & 0,53 & 29,67 \\
\hline Braunauenboden & Ap & $0-32$ & 15,77 & 30,03 & 0,53 & 24,96 \\
\hline \multirow[t]{2}{*}{ (Profil VIII) } & aMBv & $32-65$ & 15,22 & 25,32 & 0,60 & 29,19 \\
\hline & $\mathrm{aM}$ & $65-89$ & 14,54 & 24,95 & 0,58 & 28,45 \\
\hline Auengley - & Ah & $0-15$ & 28,06 & 44,10 & 0,64 & 2,21 \\
\hline Auenregosol & aMAh & $15-25$ & 25,56 & 42,71 & 0,60 & 8,88 \\
\hline \multirow[t]{2}{*}{ (Profil XI) } & $\mathrm{aM}$ & $25-50$ & 15,29 & 27,67 & 0,55 & 29,88 \\
\hline & aMGo & $50-80$ & 16,22 & 36,50 & 0,44 & 34,93 \\
\hline
\end{tabular}


de Textur von sandigem Lehm bis tonigem Schluff gekennzeichnet und spiegelt damit eine kontinuierliche Sedimentation durch Überflutung auf dem Gleithang wider.

Die Mittlere Auenterrasse kann pedologisch deutlich in eine ältere Einheit, die als maximale Bodenentwicklung eine Typische Auenbraunerde trägt, und eine jüngere Einheit mit $\mathrm{Braunauen-}$ boden gegliedert werden. In dem zur älteren Mittleren Auenterrasse gehörenden Profil VI ist der schluffig-lehmige Verwitterungshorizont (Bv) der Auenbraunerde mit einer kräftigen rötlich-braunen (7.5 YR) Färbung deutlicher ausgebildet als beim Braunauenboden (Profil VIII) der jüngeren Mittleren Auenterrasse, wo die Bodenentwicklung nur bis zur Bildung eines aMBv-Horizontes (10 YR) fortgeschritten ist. Zudem ist die Bodenart in Profil VIII sandiger, was die geringere Farbtiefe verursacht. Die pedologische Zweiteilung der Mittleren Auenterrasse stimmt somit gut mit den Ergebnissen der Holz- und Pollenanalyse überein.

Die Böden der Unteren Auenterrasse sind durch ihre Nähe zum Fluß und ihre geringe relative Höhe stark hochwassergefährdet und den Schwankungen des Sieggrundwassers ausgesetzt. Entsprechend haben sich die Böden kaum entwickelt. Bei Profil XI handelt es sich um einen Auengley-Auenregosol mit stark humosem Oberboden (zwischen $3,0 \% \mathrm{Corg}_{\mathrm{im}}$ Ah- und 1,8\% im aMAh-Horizont, Tab. 2), der in mehrere schwächer humose M-Horizonte mit ausgeprägter Feinschichtung übergeht. Der relativ hohe Humusgehalt dieses Bodens erklärt sich aus der regelmäßigen Zufuhr organischen Materials durch Hochwässer.

\subsection{Pedogene Merkmale und relatives Bodenalter}

Anhand der Ausprägung pedogener Merkmale kann die Übereinstimmung zwischen bodengenetischem Entwicklungszustand und bodenstratigraphischer Stellung überprüft werden. Als gut geeignet für die Differenzierung von Auenböden unterschiedlichen Entwicklungsgrades erweisen sich nach D. SCHRÖDER (1979: 190) neben Bodenfarbe und -gefüge besonders der Carbonat- und Kohlenstoffgehalt, der VWert, die auf Ton bezogene Kationenaustauschkapazität (KAK) sowie die Gesamtgehalte von $\mathrm{Ca}, \mathrm{Mg}$, $\mathrm{K}$, Fe und $\mathrm{Mn}$, welche mit höherem Bodenalter abnehmen. Die der Verbraunung zugrundeliegende Freisetzung zweiwertigen Eisens aus Fe-haltigen Silikaten ist analytisch insbesonders durch die pedogenen Anteile an der Gesamtfraktion nachzuweisen. Der $\mathrm{Fe}_{\mathrm{d}} / \mathrm{Fe}_{\mathrm{t}}$-Quotient steigt daher mit zunehmender Verwitterung und steigendem Alter der Böden an. Während die Werte für $F_{e_{t}}$ und $K A K / 100 \mathrm{~g}$ Ton kontinuierlich von der Parabraunerde zum Au-
engley-Auenregosol abnehmen und somit die Ergebnisse von D. SCHRÖDER bestätigen, zeigen die pedogenen Oxidanteile an der Gesamtfraktion einen umgekehrten Trend (Tab. 3). Die Vorstellung, daß der Anteil der pedogenen bzw. dithionitlöslichen Eisenverbindungen am Gesamtgehalt in den weniger entwickelten, jüngeren Auenböden geringer ist als in älteren, kann hier nicht bestätigt werden. Die bodenstratigraphische Stellung der untersuchten Auenböden wird dadurch aber nicht in Frage gestellt. Vielmehr ist anzunehmen, daß die Waldrodung im Liefergebiet infolge Erzabbau spätestens seit der Eisenzeit (WINGEN 1982) Bodenerosion ermöglichte und somit vermehrt vorverwittertes Solummaterial in den Auen abgelagert wurde. Diese Erklärung führen auch SCHIRMER \& SCHNITZLER (1980) an, die für die Auenböden am Main ab der Zeitenwende ebenfalls wesentlich höhere $\mathrm{Fe}_{\mathrm{d}}$-Anteile am Gesamteisen angeben. Allerdings stellen auch sie einen abnehmenden Trend bis zur Gegenwart fest. Offenbar führen an der Sieg gerade die eisenhaltigen Sedimente aus dem Erzabbau zu den ansteigenden und insgesamt höheren $\mathrm{Fe}_{\mathrm{d}}$-Gehalten. Der relativ hohe, durchschnittliche $\mathrm{Fe}_{\mathrm{d}} / \mathrm{Fe}_{1}$-Quotient von über 0,51 in allen Auenböden entspricht nach D. SChröder (1979: 176) den Werten von Braunerden und Parabraunerden. Daraus geht hervor, daß das kalkfreie Ausgangsmaterial aus dem Liefergebiet schon eine Eisenmobilisierung erfahren hatte, die sich dann im Verlauf der einsetzenden Bodenbildung in der Aue weiter fortsetzen konnte.

Der trotz des relativ geringen Bodenalters fortgeschrittene Entwicklungszustand der Siegauenböden - bis hin zur Ausprägung von Lessivierungsmerkmalen (Profil III) - spricht für die anthropogen beeinflußte, hohe Zufuhr an kalkfreiem, vorverwittertem Bodenmaterial im Jungholozän.

\subsection{Klassifizierung der Auenböden}

Die untersuchten Böden wurden nach der "Systematik der Böden der Bundesrepublik Deutschland" (Arbeitskreis für Bodensystematik der Deutschen Bodenkundlichen Gesellschaft 1985) in der Abteilung: Semiterrestrische Böden der Klasse der Auenböden (autochthone und allochthone Bildungen) zugeordnet, die von starken Grundwasserschwankungen und periodischen Überflutungen des Flusses gekennzeichnet sind. Die uferfernen und eingedeichten, aber im rezenten Hochwasserbett liegenden Alluvialböden zeigen z.l. keine Grundwasserbeeinflussung mehr, so daß diese Böden u. U. nunmehr eine terrestrische Entwicklung durchlaufen werden. Eine strenge Unterscheidung zwischen autochthoner und allochthoner Entstehung 
läßt sich bei den Siegauenböden wie auch bei den alluvialen Rheinböden (D. SCHRÖDER 1979, BRAUN 1991) anhand pedogener Merkmale kaum noch vornehmen. Gegen den Terminus „allochthoner Auenboden" spricht auch das Verständnis vom Boden als Verwitterungsprodukt in situ (vgl. SCHIRMER 1991b).

Entsprechend der bundesdeutschen Klassifikation, in der dem Namen des terrestrischen Bodentyps das Präfix „Auen" vorangestellt wird, wurden die Auenböden der Sieg somit als Auenparabraunerde-Auenbraunerde (Profil III), Typische Auenbraunerde (Profil VI), Braunauenboden (Profil VIII) und Auengley-Auenregosol (Profil XI) angesprochen. Damit wird auch dem Vorschlag Schirmers (1991b) gefolgt, der keine Unterscheidung zwischen autochthonen und allochthonen Bodenbildungen vorsieht.

\section{Landnutzungsgeschichte und Auengenese}

Die Entwicklung von Auen steht seit dem Neolithikum im Zusammenhang mit der Landnutzungs- und Besiedlungsgeschichte von Flußtälern. Archäologische Funde können diese Entwicklung belegen und stellen eine weitere Möglichkeit zur stratigraphischen Einordnung von Auenterrassen und ihren Böden dar.

Zahlreiche Fundstellen aus der Alt- und Jungsteinzeit liegen in unmittelbarer Nähe des Untersuchungsgebietes. Von besonderer Wichtigkeit für die zeitliche Einstufung der Hochflutlehme am Rande der weichselzeitlichen $\mathrm{Niederterrasse}$ ist die erwähnte Fundstelle der jungneolithisch/bronzezeitlichen Siedlungsstätte in Niederpleis. Die Mächtigkeit des Hochflutlehms, der auch die vorgeschichtliche Fundstelle nachträglich überdeckt hat, sowie die Erosionsdiskordanz in Profil I deuten auf eine Ablagerungszeit hin, die jünger sein muß als das Alter der Siedlungsstätte. Für die Auenlehmdecke auf der Niederterrasse, in der sich eine $\mathrm{Pa}$ rabraunerde entwickelt hat, ist aber aufgrund ihrer geomorphologischen Position ein höheres Alter zu veranschlagen als für die Ablagerungen in der Siegaue. Die im Auenlehm fein verteilte Holzkohle könnte auf bronzezeitliche Anfänge des Erzabbaus im Siegerland hindeuten, der nach WINGEN (1982) ab der Frühlatènezeit anhand von Holzkohle-Windöfen nachweisbar ist.

In der älteren Eisenzeit setzte eine stärkere Besiedlung des Untersuchungsraumes ein, die durch intensiven Ackerbau geprägt war. Der Raum Niederpleis-Buisdorf-Hennef/Geistingen entwickelte sich sogar zu einem Zentrum der mittelrheinischen Hallstattkultur (FISCHER 1974). In dieser Zeit sind die Hochflutsedimente der Oberen Auenterrasse, auf der archäologische Funde fehlen, möglicher- weise abgelagert worden. Die Auen parabraunerde-Auenbraunerde dieser ältesten Auenterrasse zeigt zudem noch deutliche Merkmale der Tonverlagerung. Auf bergbaulichen Einfluß weisen wiederum die zahlreichen Holzkohlestückchen hin. Die starke Bevölkerungszunahme in der Zeit zwischen 1000 bis $400 \mathrm{v}$. Chr. und die zunehmende Verwendung von Metallen führten zur Erschließung von Gebieten, die vorher nur dünn oder überhaupt nicht besiedelt waren. Im Siegerland boten sich aufgrund der zahlreichen, z.T. an der Erdoberfläche ausstreichenden Erzgänge aus Siderit und Brauneisenstein ideale Voraussetzungen für den sich nun auf dem Höhepunkt befindlichen eisenzeitlichen Erzabbau, der intensive Waldrodungen für die Verhüttung nach sich zog. Dadurch gelangte vermehrt lithogenes und pedogenes Material in die Siegaue, so daß die Typische Auenbraunerde der älteren Mittleren Auenterrasse eine kräftige, hellbraune Färbung aufweist. Anhand der pedologischen Kriterien und des auf $2210 \pm 70 \mathrm{BP}$ datierten Holzfundes muß geschlossen werden, daß der Hochflutlehm dieser Auenterrasse seit der Latènezeit abgelagert wurde.

Da das Abholzen der Wälder im Einzugsgebiet zur Energiegewinnung allerdings in uneingeschränkter Weise erfolgte, führte der Raubbau an der Natur zur „ersten Siegerländer Energiekrise“ (WINGEN 1982). Sie dürfte ein wichtiger Grund für das Ende der Eisenverhüttung in der Spätlatènezeit gewesen sein. Während der Römischen Kaiserzeit kam es aufgrund der Nachbarschaft zu den Zentren Bonn und Köln zu einem Rückgang der Besiedlungsdichte im Siegerland, der bis zum 9. Jahrhundert andauerte. Möglicherweise besteht zwischen der älteren und jüngeren Mittleren Auenterrasse ein zeitlicher Hiatus, der durch die Wiederbewaldung des Einzugsgebietes während des 1 . bis 9. Jahrhunderts und den Rückgang der Erosion hervorgerufen wurde.

Die wiederbewaldeten Gebiete wurden im Zuge der fränkischen Binnenkolonisation ab dem 9. Jahrhundert erneut gerodet. Unter dem Einfluß dieses Siedlungsausbaus - Siegen wird 1079 erstmals urkundlich erwähnt - setzte die Eisenverhüttung im Siegerland während des 10. bis 13. Jahrhunderts wieder ein (WINGEN 1982). Im Hoch- und Spätmittelalter wurden Phasen verstärkter Rodungsaktivität immer wieder durch Wanderbewegungen und Seuchen unterbrochen, die zeitweise zur Entvölkerung ganzer Landstriche führten. Die Verbuschung aufgegebener Ackerflächen in der Siegaue kann anhand der Pollenanalyse aufgezeigt werden. Das auf $800 \pm 50$ BP datierte Holzstück, der wieder einsetzende Bergbau sowie die Pollenund Keramikfunde deuten daher für den Braunauenboden der jüngeren Mittleren Au- 
enterrasse eine mittelalterliche bis frühneuzeitliche Entstehung an.

Die Verlagerung der Sieg auf der Unteren Auenterrasse ist anhand genauer Preußischer Urkatasterpläne (Katasteramt Siegburg 1826/1827) gut rekonstruierbar: Während sie offenbar ihren geraden Lauf zwischen Allner und Kaldauen seit mehreren Jahrhunderten nur wenig geändert hat, erlangte der Mäanderbogen zwischen Kaldauen und Wolsdorf in weniger als 30 Jahren am Ende des 18. Jahrhunderts seine aktuelle Größe. Somit ist die Untere Auenterrasse mit dem auf ihr entwickelten $\mathrm{Au}$ engley/Auenregosol nicht älter als 200 Jahre. Der Abbau von Buntmetallen im Siegerland erlebte nach RöHrig (1992) mit der Schürfung von Bleiglanz, Kobaltblüte, Kupferkies und Zinkblende noch vor hundert Jahren eine Blütezeit. Die letzte Grube wurde erst 1966 stillgelegt. Der starke Einfluß des Bergbaus auf die Auengenese seit der Eisenzeit zeigt sich auch am Gesamtgehalt von Blei, Kobalt, Kupfer und Zink, der in jedem Horizont aller untersuchten Auenböden den von KLOKE (1985) angegebenen geogenen Grundgehalt an Schwermetallen von Böden übersteigt (hier nicht dargestellt). Die höchsten Werte wurden dabei im Oberboden des Auengleys gemessen, der als einziger Auenboden seit dem Bau des Schutzdeiches (Archiv des Rhein-Sieg-Kreises 1976) periodisch überflutet wird.

\section{Schlußfolgerung}

In der Siegaue bei Hennef entstand eine viergliedrige Terrassenabfolge mit einer Bodenchronosequenz jungholozänen Alters. Anhand der vorgestellten Ergebnisse geomorphologisch-sedimentologischer sowie bodenkundlicher Untersuchungen kann die Bildung der Auensedimente auf den Siegterrassen bestimmten Phasen erhöhter Bergbautätigkeit im Einzugsgebiet zugeordnet werden. Die Ab- bzw. Umlagerung der Siegschotter mag zwar einem übergeordneten klimatischen Impuls folgen (SCHIrmer 1983a); die Sedimentation der Auenlehme wird aber bereits seit der Bronze/Eisenzeit verstärkt vom Menschen beeinflußt.

\section{Danksagung}

Wir danken der Deutschen Forschungsgemeinschaft, die im Rahmen des Sonderforschungsbereiches 350 „Wechselwirkungen kontinentaler Stoffsysteme und ihre Modellierung" das Teilprojekt B3-1 „Transport und Sedimentation/Umlagerung von Feststoffen sowie Reliefbildung auf flußnahen Auenböden der Sieg" gefördert hat. Unser Dank gilt auch Herrn Prof. Dr. W. Schirmer, Abt. Geologie des Geographischen Instituts der Universität Düsseldorf, für eine gemeinsame Geländebegehung am 24. Februar 1992 und Frau Dr. U. Leßmann-Schoch, Institut für Bodenkunde der Universität Bonn, für die Hilfestellung bei der Pollenanalyse. Die Radiocarbondatierungen übernahm dankenswerterweise Herr Dr. P. Becker-Heidmann, Institut für Bodenkunde der Universität Hamburg.

\section{Schriftenverzeichnis}

Arbeitskreis für Bodensystematik der Deutschen Bodenkundlichen Gesellschaft (Hrsg.) (1985): Systematik der Böden der Bundesrepublik Deutschland. - Mitteilgn. Dtsch. Bodenkundl. Gesellsch., 44: 91 S.; Göttingen (Deutsche Bodenkundliche Gesellschaft).

Archiv des Rhein-Sieg-Kreises (1976): Akten des Landratsamtes Siegkreis von 1891-1934. - Quellen zur Geschichte des Rhein-Sieg-Kreises, 3: 266-267, Akten-Nr. 1978, 2331, 2495, 2637; Siegburg (Respublica).

Behl, T. \& Krämer, R. (1992): Die hydrologischen Verhältnisse im Einzugsgebiet der geplanten Grundwassergewinnungsanlage im Hennefer Siegbogen. - II + 15 S., 6 Abb., 7 Tab., 7 Krt.; Siegburg (Wahnbachtalsperrenverband).

Braun, P. (1991): Gefügeverfestigung in Auenböden des Mittelrheins. - Bonner Bodenkundl. Abh., 3: 354 S., 204 Abb., 60 Tab.; Bonn (Selbstverlag).

BrunnaCker, K. (1978): Der Niederrhein im Holozän. Fortschr. Geol. Rheinld. u. Westf., 28: 399-440, 14 Abb., 4 Tab., 1 Taf.; Krefeld.

Buch, M. W. (1988): Spätpleistozäne und holozäne fluviale Geomorphodynamik im Donautal zwischen Regensburg und Straubing. - Regensburger Geogr. Schr., 21 (I + II): 197 S., 55 Abb., 14 Fotos, 14 Krt., 6 Tab.; Regensburg (Selbstverlag).

Dalchow, C. (1989): Vorlesungsauswertungen Heinrich Rohdenburg: Geoökologie - Geomorphologie. - 201 S., 106 Fig., 8 Tab.; Cremlingen-Destedt (Catena-Verlag).

Fischer, H. (1974): Die frühe Besiedlung des GeistingenHennefer Raumes. - Beitr. z. Gesch. d. Gemeinde Hennef-Sieg, 5: 9-23, 7 Abb.; Hennef.

Gramsch, H.-J. (1978): Die Entwicklung des Siegtales im jüngsten Tertiär und im Quartär. - Bochumer Geogr. Arb., 31: 188 S., 35 Abb., 30 Tab.; Paderborn (Schöningh).

Hiller, A., LitT, T. \& Eissmann, L. (1991): Zur Entwicklung der jungquartären Tieflandstäler im Saale-Elbe-Raum unter besonderer Berücksichtigung von $\mathrm{C}^{14}$-Daten. Eiszeitalter u. Gegenwart, 41: 26-46, 12 Abb., 1 Tab.; Hannover.

Kalis, A. J. \& Meurers-Balke, J. (1994): Die Vegetationsgeschichte. - In: BRunotTe, E., IMMENDORF, R. \& SCHLimm, R. (Hrsg.): Die Naturlandschaft und ihre Umgestaltung durch den Menschen, Kölner Geogr. Arb., 63, S. 1422, 2 Abb., 1 Tab.; Köln (Selbstverlag).

Katasteramt Siegburg (1826/1827): Preußische Urkatasterkarten 1:1250 u. 1:1500; 1863 korrigiert, Regierungsbezirk Köln, Rhein-Sieg-Kreis, Gemeinden Buisdorf, Geistingen, Altenbödingen, Striefen und Lauthausen; Siegburg (Bauamt Allner).

KLOKE, A. (1985): Richt- und Grenzwerte zum Schutz des Bodens vor Überlastungen mit Schwermetallen. - Forschungen zur Raumentwicklung, 14: 13-24, 4 Abb., 7 Tab.; Bonn. 
KlostermanN, J. (1992): Das Quartär der Niederrheinischen Bucht. Ablagerungen der letzten Eiszeit am Niederrhein. - 200 S., 30 Abb., 8 Tab., 2 Taf.; Krefeld (Geol. Landesamt NRW).

KNuth, H. (1923): Die Terrassen der Sieg von Siegen bis zur Mündung. - Beitr. zur Landeskunde der Rheinlande, 4: 112 S., 2 Abb., 5 Tab.; Leipzig (Akademische Verlagsgesellschaft).

Kremer, B. P. \& Caspers, N. (1986): Die Siegniederung. Rheinische Landschaften, 27: 35 S., 43 Abb.; Neuss (Neusser Druckerei und Verlag).

Marschall, A., NARR, K. J. \& v. Uslar, R. (1954): Die vorund frühgeschichtliche Besiedlung des Bergischen Landes. - Zeitschr. d. Berg. Geschichtsvereins, 73: 272 S., 150 Abb., 3 Beil.; Neustadt an der Aisch (Ph. C. W. Schmidt).

RöHrig, H. (1992): Der kurze Goldrausch. Auf den Spuren des Bergbaus an der Sieg. - Rhein-Sieg-Anzeiger, Sonderdruck: 11 S., 19 Abb.; Köln (M. DuMont Schauberg).

SCHIRMER, W. (1983a): Die Talentwicklung an Main und Regnitz seit dem Hochwürm. - Geol. Jb., A 71 : 11-43, 9 Abb.; Hannover.

- (1983b): Symposium „Franken“: Ergebnisse zur holozänen Talentwicklung und Ausblick. - Geol. Jb., A 71: 355-370, 2 Abb.; Hannover.

- (1991a): Bodensequenz der Auenterrassen des Maintals. - Bayreuther Bodenkundl. Ber., 17 (Supplementband NO-Bayern): 153-186, 9 Abb.; Bayreuth. - (1991b): Zur Nomenklatur der Auenböden mitteleuropäischer Flußauen. - Mitteilgn. Dtsch. Bodenkundl. Gesellsch., 66: 839-842, 1 Abb.; Oldenburg. - \& Schnitzler, J. (1980): Eisengehalte der Auenböden am Main. - In: Schirmer, W. (Hrsg.): Exkursionführer zum Symposium Franken. Holozäne Talentwicklung - Methoden und Ergebnisse, S. 66-69, 2 Abb.; Düsseldorf (Internationale Union für Quartärforschung).
SCHRÖDER, D. (1979): Bodenentwicklung in spätpleistozänen und holozänen Hochflutlehmen des Niederrheines. - Habilitationsschrift Bonn: 296 S., 63 Abb., 14 Tab., 56 Tab.; Bonn.

SCHRÖDER, E. (1965): Zur Talgeschichte der Unteren Sieg. - Decheniana, 118: 41-45, 2 Abb; Bonn.

- (1969): Alter und Entstehung der rechtsrheinischen Trogfläche zwischen Agger und Sieg. - Decheniana, 122: 21-29, 3 Abb.; Bonn.

Thoste, V. (1974): Die Niederterrassen des Rheins vom Neuwieder Becken bis in die Niederrheinische Bucht. - Diss. Univ. Köln: 130 S., 31 Abb., 6 Tab.; Köln.

Uduuft, H. (1977): Geologische Karte von Nordrhein-Westfalen 1:25 000. Erläuterungen zu Blatt 5209 Siegburg. - 2. Aufl., VI + 76 S., 2 Abb., 6 Tab., 1 Taf.; Krefeld (Geol. Landesamt NRW).

WARSTAT, M. (1981): Erläuterungen zur Bodenkarte 1:5000, Verfahren: Hennef, Rhein-Sieg-Kreis. - 6 S., 8 Krt., 1 Leg.; Krefeld (Geol. Landesamt NRW).

- (1985): Erläuterungen zur Bodenkarte "Wiesengut" im Maßstab 1:2000. - 8 S. + VII, 4 Abb., 1 Krt.; Krefeld (Geol. Landesamt NRW).

Wildhagen, H. \& Meyer, B. (1972): Holozäne Boden-Entwicklung, Sediment-Bildung und Geomorphogenese im Flußauen-Bereich des Göttinger Leinetal-Grabens. 2: Die Auenlehm-Decken des Subatlantikums. - Göttinger Bodenkundl. Ber., 21: 77-158, 6 Abb., 9 Krt., 5 Tab.; Göttingen.

Wingen, H. (1982): Energie aus dem Hauberg. - 113 S., 46 Abb., 7 Tab.; Siegen (Höpner).

Wisplinghoff, E. (1964): Urkunden und Quellen zur Geschichte der Stadt und Abtei Siegburg. - Bd. I: 587588, Urkunde Nr. 549; Siegburg (Respublica).

Manuskript eingegangen am 11. 11. 1994 